\title{
CYCLIC SYMMETRY ON COMPLEX TORI AND BAGNERA-DE FRANCHIS MANIFOLDS
}

\author{
FABRIZIO CATANESE
}

\begin{abstract}
We describe the possible linear actions of a cyclic group $G=\mathbb{Z} / n$ on a complex torus, using the cyclotomic exact sequence for the group algebra $\mathbb{Z}[G]$. The main application is devoted to a structure theorem for Bagnera-De Franchis Manifolds, but we also give an application to hypergeometric integrals.
\end{abstract}

\section{Contents}

Introduction

1. An exact sequence in factorial rings

2. An arithmetic application

3. $\quad R(n):=\mathbb{Z}\left[C_{n}\right]=\mathbb{Z}[x] /\left(x^{n}-1\right)$-modules which are torsion free Abelian groups.

4. Fully ramified cyclic coverings of the projective line and associated Hodge structures

5. Structure Theorem for Bagnera-De Franchis Manifolds

6. The intersection product for the homology of fully ramified cyclic coverings of the line

References

Date: February 6, 2019.

AMS Classification: 14K99, 14D99, 32Q15, 32M17, 32Q57, 11A07, 11R18, $13 \mathrm{C} 05$.

The present work took place in the framework of the ERC Advanced grant $\mathrm{n}$. 340258, 'TADMICAMT' . 


\section{INTRODUCTION}

Classically, the word 'hyperelliptic' was used for two different ways of generalizing the class of elliptic curves, i.e. the complex tori of dimension 1.

Hyperelliptic curves are defined to be the curves who admit a map to $\mathbb{P}^{1}$ of degree 2, and are not Hyperelliptic Varieties according to the definition of the French school of Appell, Humbert, Picard, Poincaré. The French school defined the Hyperelliptic Varieties as those smooth projective varieties whose universal covering is biholomorphic to $\mathbb{C}^{g}$ (in particular the Abelian varieties are in this class). For $g=1$ these are just the elliptic curves, whereas a prize, the Bordin prize, was offered for those mathematicians who would achieve the classification of the Hyperelliptic varieties of dimension 2. Enriques and Severi were awarded the Prize in 1907 ([EnrSev09]), but they withdrew their first paper after discussion with De Franchis (replacing it with a second one); Bagnera and De Franchis were awarded the Bordin Prize in 1909, they gave a simpler proof ([BdF08] ) apart of a small gap; perhaps for this reason we prefer to call Bagnera-De Franchis surfaces the Hyperelliptic surfaces which are not Abelian surfaces.

Kodaira Kod66 showed that if we take the wider class of compact complex manifolds of dimension 2 whose universal covering is $\mathbb{C}^{2}$, then there are other non algebraic and non Kähler surfaces, called nowadays Kodaira surfaces.

Based on Kodaira's work, Iitaka conjectured that if a compact Kähler Manifold $X$ has universal covering biholomorphic to $\mathbb{C}^{g}$, then necessarily $X$ is a quotient $X=T / G$ of a complex torus $T$ by the free action of a finite group $G$ (which we may assume to contain no translations). The conjecture by Iitaka had been proven in dimension 2 by Kodaira, and was much later proven in dimension 3 by Campana and Zhang CamZha05. Whereas it was shown in CHK13 that, if the abundance conjecture holds, then a projective smooth variety $X$ with universal covering $\mathbb{C}^{n}$ is a Hyperelliptic variety according to the following definition.

Definition 0.1. A Hyperelliptic Manifold $X$ is defined to be a quotient $X=T / G$ of a complex torus $T$ by the free action of a finite group $G$ which contains no translations.

We say that $X$ is a Hyperelliptic Variety if moreover the torus $T$ is projective, i.e., it is an Abelian variety $A$.

If the group $G$ is a cyclic group $\mathbb{Z} / n$, then such a quotient is called ([BCF15], Cat15]) a Bagnera-De Franchis manifold (in dimension $g=$ $2, G$ is necessarily cyclic, whereas in dimension $g \geq 3$ the only examples with $G$ non Abelian have $G=D_{4}$ and were classified in [CD18-2] (for us $D_{4}$ is the dihedral group of order 8). 
Indeed, (see for instance CD17]) every Hyperelliptic Manifold is a deformation of a Hyperelliptic Variety, so that a posteriori the two notions are related to each other, in particular the set of underlying differentiable manifolds is the same.

By the so-called Bieberbach's third theorem Bieb11, Bieb12] concerning the finiteness of Euclidean cristallographic groups, Hyperelliptic manifolds of a fixed dimension $g$ belong to a finite number of families. We give in this paper an explicit boundedness result for the case of Bagnera-De Franchis Manifolds, for which $G$ is a finite cyclic group (Theorem 5.1).

The theory of Bagnera-De Franchis Manifolds was introduced in [BCF15], and also expounded in Cat15 (following ideas introduced first in CatCil93) and our main motivation here was to expand and improve the presentation given there, whereas we refer the reader to [Dem16] for a classification of Bagnera-De Franchis Manifolds of low dimension.

In order to do so, it is necessary to treat linear actions of a finite group $G$ on a complex torus, and we do this here for the case of a cyclic group. The subtle point is to describe these actions not only up to isogeny, but determining explicit the torsion subgroups involved in these isogenies. The starting point is that a linear action of a group $G$ on a complex torus consists in two steps:

1) viewing $\Lambda:=H_{1}(T, \mathbb{Z})$ as a $\mathbb{Z}[G]$-module

2) choosing an appropriate Hodge decomposition on $\Lambda \otimes \mathbb{C}:=\Lambda \otimes_{\mathbb{Z}} \mathbb{C}$

$$
\Lambda \otimes \mathbb{C}=H^{1,0} \oplus \overline{H^{1,0}}
$$

which is invariant for the group action (i.e., $H^{1,0}$ is a $G$-invariant subspace).

While 2) uses, for $G$ cyclic, just the eigenspace decomposition, 1) requires us to explain in detail some elementary and mostly well known facts about the group algebra of a cyclic group $G, \mathbb{Z}[G]=\mathbb{Z}[x] /\left(x^{n}-1\right)$. This is derived in section 3 from an elementary generalization of the Chinese remainder theorem (Theorem 1.1), concerning quotients of factorial rings by principal ideals (these appear naturally in the intersection theory of divisors), and from some classical results about resultants of cyclotomic polynomials, explained in section 2 . The interesting result for our purposes is Proposition 3.3 .

The first application that we give is related to hypergeometric integrals: we calculate explicitly the homology of a cyclic covering of degree $n$ of the projective line $\mathbb{P}^{1}$ as a $\mathbb{Z}[\mathbb{Z} / n]$-module, under the assumption that there is a point of full ramification. The description is particularly nice for the case where there are two points of full ramification, Theorem 4.3 shows that we have a direct sum of certain cyclic modules naturally associated to the ramification indices. 


\section{Theorem 4.3}

Assume that we have a cyclic covering $f: C \rightarrow \mathbb{P}^{1}$ with group $\mathbb{Z} / n$ and with two points of full ramification.

Then, if the order of the inertia groups are $n, r_{1}, \ldots, r_{k}, n$, the $\mathbb{Z}[x]$ module $H_{1}(C, \mathbb{Z})$ is a direct sum of cyclic modules,

$$
H_{1}(C, \mathbb{Z})=\oplus_{1}^{k} \mathbb{Z}[x] /\left(1+x^{n / r_{j}}+x^{2 n / r_{j}}+\cdots+x^{\left(r_{j}-1\right) n / r_{j}}\right) .
$$

In the final section 6 we determine also explicitly the intersection product for the first homology group $H_{1}(C, \mathbb{Z})$.

We pose the question of finding a simple description in the general case.

Section 5 is devoted to the second and main application, namely, the algebraic description of Bagnera-De Franchis Manifolds with group $\mathbb{Z} / n$. The main result is Theorem 5.1 .

\section{Theorem 5.1}

A Bagnera-De Franchis Manifold with group $G=\mathbb{Z} / n$ is completely determined by the following data:

(1) the datum of torsion free $R_{d}$-modules $\Lambda_{d}$ of finite rank, for all $d \mid n$, such that $\Lambda_{1} \neq 0$ and with $\Lambda_{1}, \Lambda_{2}$ of even rank;

(2) the datum of a finite subgroup $\Lambda^{0} \subset A^{\prime}:=\oplus_{d \mid n} A_{d}$, where $A_{d}:=$ $\Lambda_{d, \mathbb{R}} / \Lambda_{d}$

(3) an element $\beta_{1} \in A_{1}$ generating a subgroup $\left\langle\beta_{1}\right\rangle$ of order exactly $n$, such that:

(4) (A) $\Lambda^{0}$ is stable for multiplication by the element $x$ of the subring $R(n) \subset R^{\prime}(n):=\oplus_{d \mid n} R_{d}$, and

(5) (B) $\Lambda^{0} \cap A_{d}=0 \forall d \mid n$,

(6) (C) the projection of $\Lambda^{0}$ into $A_{1}$ intersects the subgroup $\left\langle\beta_{1}\right\rangle$ only in 0 ;

(7) the datum of a complex structure on each $\Lambda_{d} \otimes \mathbb{C}$, i.e., a Hodge decomposition

$$
\Lambda_{d} \otimes \mathbb{C}=V(d) \oplus \overline{V(d)},
$$

which allows to decompose $V(d)=\oplus_{j<d,(j . d)=1} V_{j}$ as a direct sum of eigenspaces for the action $\alpha$ of $x$.

(8) The properties (A) and (B) imply that $\Lambda^{0} \subset \oplus_{d \mid n}\left(\frac{\Phi_{d}}{Q_{n}} \Lambda_{d}\right) / \Lambda_{d}$, hence, in particular, the number of such subgroups $\Lambda^{0}$ is finite.

Throughout the paper we have been trying to illustrate the concepts introduced, or discussed, via many concrete examples.

\section{An EXACT SEQUENCE IN FACTORIAL RINGS}

Theorem 1.1. Let $\mathcal{O}$ be a factorial ring, and assume that we have an integer $k \geq 2$ and elements $f_{1}, \ldots, f_{k} \in \mathcal{O}$, such that

(1) $f_{i}$ is not a unit

(2) for $i \neq j, f_{i}$ and $f_{j}$ are relatively prime. 
Then we have a natural exact sequence

$$
0 \rightarrow R:=\mathcal{O} /\left(f_{1} f_{2} \ldots f_{k}\right) \rightarrow \oplus_{1}^{k} \mathcal{O} /\left(f_{i}\right) \rightarrow \oplus_{i<j} \mathcal{O} /\left(f_{i}, f_{j}\right) \rightarrow 0
$$

where, setting $R_{i}:=\mathcal{O} /\left(f_{i}\right), R_{i, j}:=\mathcal{O} /\left(f_{i}, f_{j}\right)$ for $i \neq j$,

(3) $R \rightarrow \oplus_{1}^{k} R_{i}$ is induced by the natural surjections $R \rightarrow R_{i}=\mathcal{O} /\left(f_{i}\right)$, and where

(4) $\left(a_{i}\right) \in \oplus_{1}^{k} R_{i} \mapsto\left(a_{i}-a_{j}\right) \in \oplus_{i<j} \mathcal{O} /\left(f_{i}, f_{j}\right)$.

Remark 1.2. Observe that the hypothesis that $f_{i}$ is not a unit is not really needed, since, if $f_{i}$ is a unit, then $R_{i}=0=R_{i, j} \forall j$, and we have the same exact sequence as the one corresponding to the set obtained from the set $\left\{f_{1}, \ldots, f_{k}\right\}$ by deleting $f_{i}$.

The proof follows essentially by induction from the standard special case $k=2$ :

Lemma 1.3. Assume either that

(I) $\mathcal{O}$ is a factorial ring, and $f, g \in \mathcal{O}$ are relatively prime elements.

Or assume that

$(I I) \mathcal{O}$ is any ring, the ideal $(f)$ is prime, $g \notin(f)$.

Then we have a natural exact sequence

$$
0 \rightarrow R:=\mathcal{O} /(f g) \rightarrow \mathcal{O} /(f) \oplus \mathcal{O} /(g) \rightarrow \mathcal{O} /(f, g) \rightarrow 0 .
$$

Proof. We first prove the exactness of

$$
\mathcal{O} \rightarrow \mathcal{O} /(f) \oplus \mathcal{O} /(g) \rightarrow \mathcal{O} /(f, g) \rightarrow 0 .
$$

Surjectivity is obvious, whereas if $(a(\bmod f), b(\bmod g))$ maps to 0 , then

$a-b \in(f, g) \leftrightarrow \exists \alpha, \beta \in \mathcal{O}, a-b=\alpha f+\beta g \leftrightarrow a-\alpha f=b+\beta g:=c$.

But then $c-a \in(f), c-b \in(g)$, proving exactness in the middle.

Finally, $c \in \mathcal{O} \mapsto(0,0)$ if and only if $c \in(f) \cap(g)$.

In case (I), by unique factorization and since $f, g$ are relatively prime, $c$ is divisible by $f g$, hence the kernel of the first homomorphism is the principal ideal $(f g)$.

In case (II), $c \in(g)$ implies the existence of $d$ such that $c=d g$. Since $c \in f$, and $(f)$ is prime, then necessariy either $g \in(f)$, or $d \in(f)$. The first possibility is excluded by our assumption, therefore there exists $e \in \mathcal{O}$ with $d=e f$, hence $c=e f g$, and we are done.

Corollary 1.1. Let $\mathcal{O}$ be a factorial ring, and assume that we have an integer $k \geq 2$ and elements $f_{1}, \ldots, f_{k} \in \mathcal{O}$, such that

(1) $f_{i}$ is not a unit

(2) for $i \neq j, f_{i}$ and $f_{j}$ are relatively prime.

Then, setting $F:=f_{1} f_{2} \ldots f_{k}$, the cokernel $N$ of the following exact sequence: 


$$
0 \rightarrow R:=\mathcal{O} /(F) \rightarrow \oplus_{1}^{k} \mathcal{O} /\left(f_{i}\right) \rightarrow N \rightarrow 0
$$

has a filtration

$$
0:=N_{0} \subset N_{1} \subset \cdots \subset N_{k-1}=N
$$

such that $N_{i} / N_{i-1} \cong \mathcal{O} /\left(f_{i}, f_{i+1} \ldots f_{k}\right)$.

Proof. Observe first of all that $R \rightarrow \oplus_{1}^{k} R_{i}$ is an inclusion, since our elements $f_{i}$ are relatively prime.

We prove now the main assertion by induction on $k$, the case $k=2$ being the content of lemma 1.3 .

Set $g:=f_{2} \ldots f_{k}$, and observe that $F=f_{1} g$ : by lemma 1.3 we have an exact sequence

$$
0 \rightarrow R=\mathcal{O} /(F) \rightarrow \mathcal{O} /\left(f_{1}\right) \oplus \mathcal{O} /\left(f_{2} \ldots f_{k}\right) \rightarrow \mathcal{O} /\left(f_{1}, f_{2} \ldots f_{k}\right) \rightarrow 0
$$

By induction we have an exact sequence

$$
0 \rightarrow R^{\prime}:=\mathcal{O} /\left(f_{2} \ldots f_{k}\right) \rightarrow \oplus_{2}^{k} \mathcal{O} /\left(f_{i}\right) \rightarrow N^{\prime} \rightarrow 0,
$$

and a filtration $N_{0}^{\prime} \subset \cdots N_{k-1}^{\prime}=N^{\prime}$ with the desired properties.

Hence we have inclusions

$$
0 \rightarrow R \rightarrow R_{1} \oplus R^{\prime} \rightarrow \oplus_{1}^{k} R_{i}
$$

and, defining $N_{1}:=\mathcal{O} /\left(f_{1}, f_{2} \ldots f_{k}\right)$, we have $N^{\prime}=N / N_{1}$, and it suffices to define $N_{i}$, for $i \geq 2$, to be the inverse image of $N_{i-1}^{\prime}$ inside $N$.

Lemma 1.4. Let $f, h, g \in \mathcal{O}$ and assume either that

(i) the ring $\mathcal{O}$ is factorial, and $f, g$ are relatively prime, or

(ii) the ideal $(f)$ is a prime ideal, and $g \notin(f)$.

Then we have the exact sequence, where the first map is given by multiplication by $g$ :

$$
0 \rightarrow \mathcal{O} /(f, h) \rightarrow \mathcal{O} /(f, g h) \rightarrow \mathcal{O} /(f, g) \rightarrow 0 .
$$

Proof. Set $A:=\mathcal{O} /(f)$.

Then we have an exact sequence

$$
A \rightarrow A /(g h) \rightarrow A /(g) \rightarrow 0,
$$

and the kernel of the first map is the principal ideal generated by $h$, since

$$
\{\phi \in A \mid \phi g \in(g h)\}=\{\phi \mid \exists \psi \phi g=g h \psi\}=\{\phi \mid \exists \psi \phi=h \psi\},
$$

because $g$ is not a zero divisor in $A$. 
Lemma 1.5. Let $\mathcal{O}$ be a factorial ring, and assume that we have an integer $k \geq 2$ and elements $f_{1}, \ldots, f_{k} \in \mathcal{O}$, such that

(1) $f_{i}$ is not a unit

(2) for $i \neq j, f_{i}$ and $f_{j}$ are relatively prime.

Then $\mathcal{O} /\left(f_{1}, f_{2} \ldots f_{k}\right)$ has a filtration whose graded quotient is

$$
\oplus_{j=2}^{k} \mathcal{O} /\left(f_{1}, f_{j}\right)
$$

Proof. Apply lemma 1.4 to $f:=f_{1}, h:=f_{k}$, and $g:=f_{2} \ldots f_{k-1}$, and use induction.

\section{Proof of Theorem 1.1}

We first of all observe that the map $\oplus_{1}^{k} R_{i} \rightarrow M:=\oplus_{i<j} R_{i, j}$ factors through the quotient $N$.

We have shown that $N$ has a filtration whose associated graded ring is exactly isomorphic to $\oplus_{i<j} R_{i, j}$, and from this we shall derive an isomorphism of $N$ with $\oplus_{i<j} R_{i, j}$.

In fact, by induction, the homomorphism $N \rightarrow M$ induces an isomorphism $N^{\prime} \cong \oplus_{i<j, i, j \geq 2} R_{i, j}:=M^{\prime}$.

Moreover, by the definition of the map, $R_{1} \oplus R^{\prime}$ maps to zero inside $M^{\prime}$.

Hence it suffices to show that $\left(R_{1} \oplus R^{\prime}\right) / R=N_{1}$ maps isomorphically to $M_{1}:=\oplus_{j \geq 2} R_{1, j}$, and this follows again by corollary 1.5 observing that the homomorphism preserves the corresponding filtrations on both modules $M_{1}, N_{1}$ and that the homomorphism induces an isomorphism of associated graded modules: since, by induction (changing the order of the summands), for each $j, N_{1}$ surjects onto $R_{1, j}$.

Hence this homomorphism induces an isomorphism $N_{1} \cong M_{1}$ and the proof is finished.

We record here a result shown in the course of the proof of Theorem

Corollary 1.2. Let $\mathcal{O}$ be a factorial ring, and assume that we have an integer $k \geq 2$ and elements $f_{1}, \ldots, f_{k} \in \mathcal{O}$, such that

(1) $f_{i}$ is not a unit

(2) for $i \neq j, f_{i}$ and $f_{j}$ are relatively prime.

Then the $\mathcal{O}$-module $\mathcal{O} /\left(f_{1}, f_{2} \ldots f_{k}\right)$ is isomorphic to

$$
\oplus_{j=2}^{k} \mathcal{O} /\left(f_{1}, f_{j}\right) \text {. }
$$

\section{An ARIthmetic APPLiCATION}

In this section we consider the factorial $\operatorname{ring} \mathcal{O}:=\mathbb{Z}[x]$, set

$$
Q_{n}:=x^{n}-1, \quad R(n):=\mathbb{Z}[x] /\left(Q_{n}\right) .
$$

We have, setting $\mu_{n}:=\left\{\zeta \in \mathbb{C} \mid \zeta^{n}=1\right\}$,

$$
Q_{n}(x)=\prod_{\zeta \in \mu_{n}}(x-\zeta),
$$


and we have an irreducible decomposition in $\mathbb{Z}[x]$

$$
Q_{n}(x)=\Pi_{d \mid n} \Phi_{d}(x),
$$

where $\Phi_{d}(x)$ is the d-th cyclotomic polynomial

$$
\Phi_{d}(x)=\prod_{\zeta \in \mu_{n}, \operatorname{ord}(\zeta)=d}(x-\zeta) .
$$

We have (see Lang, page 280),

$$
\Phi_{d}(x)=\Pi_{d^{\prime} \mid d}\left(x^{d / d^{\prime}}-1\right)^{\mu\left(d^{\prime}\right)},
$$

where $\mu\left(d^{\prime}\right)$ is the Möbius function, such that

- $\mu\left(d^{\prime}\right)=0$ if $d^{\prime}$ is not square-free

- $\mu(1)=1$

- $\mu\left(p_{1} \ldots p_{r}\right)=(-1)^{r}$, if $p_{1}, \ldots, p_{r}$ are distinct primes.

Definition 2.1. Define the cyclotomic ring as $R_{d}:=\mathbb{Z}[x] /\left(\Phi_{d}\right)$, and, for integers $d \neq m, R_{d, m}:=\mathbb{Z}[x] /\left(\Phi_{d}, \Phi_{m}\right)$.

Example 2.2. Consider the polynomial $Q_{6}=x^{6}-1=\left(x^{3}-1\right)\left(x^{3}+\right.$ $1)=(x-1)\left(x^{2}+x+1\right)(x+1)\left(x^{2}-x+1\right)=\Phi_{1} \Phi_{3} \Phi_{2} \Phi_{6}$.

We choose now $d=3, m=6$ : then, since $\Phi_{6}=\Phi_{3}-2 x$, and $2\left(x^{2}+\right.$ $x+1)-2 x(x+1)=2$, we obtain that

$$
\left(\Phi_{3}, \Phi_{6}\right)=\left(x^{2}+x+1,2 x\right)=\left(x^{2}+x+1,2\right) .
$$

Hence $R_{3,6}=\mathbb{Z} / 2[x] /\left(x^{2}+x+1\right)=\mathbb{F}_{4}$.

While $\mathbb{Z} /\left(\left(\Phi_{3}, \Phi_{6}\right) \cap \mathbb{Z}\right)=\mathbb{Z} / 2$.

Note that $r:=\operatorname{Res}_{x}\left(\Phi_{3}, \Phi_{6}\right)$ equals, by the interpolation formula, if $\zeta$ is a primitive third root of $1, r=\left(\zeta^{2}-\zeta+1\right)\left(\zeta-\zeta^{2}+1\right)=(-2 \zeta)\left(-2 \zeta^{2}\right)=$ $4=\left|R_{3,6}\right|$.

Instead, easily we get $R_{1,3}=\mathbb{Z} / 3, R_{1,6}=0, R_{1,2}=\mathbb{Z} / 2, R_{2,3}=0$, $R_{2,6}=\mathbb{Z} / 3$.

Observe now in general that, since $\Phi_{d}, \Phi_{m}$ are monic polynomials, and both irreducible, their resultant is a non zero integer $r=r_{d, m}$, such that, if

$$
\mathbb{Z} /\left(\left(\Phi_{m}, \Phi_{d}\right) \cap \mathbb{Z}\right)=\mathbb{Z} /\left(\beta_{d, m}\right),
$$

then $\beta_{d, m} \mid r_{d, m}$ and the two numbers have the same radical.

It is easy to see that $R_{d, m}=0$ if $d, m$ are relatively prime: since then in the quotient we have $x^{m} \equiv 1, x^{d} \equiv 1 \Rightarrow(x-1) \equiv 0$, hence $m \equiv 0 \Rightarrow R_{d, m}=0$.

It is straightforward to calculate the discriminant of $Q_{n}=x^{n}-1$ as the resultant of $Q_{n}$ and its derivative: $\operatorname{Disc}\left(Q_{n}\right)=n^{n}$.

However, up to $\pm 1, \operatorname{Disc}\left(Q_{n}\right)=\prod_{0 \leq i<j \leq n-1}\left(\epsilon_{n}^{i}-\epsilon_{n}^{j}\right)$, where $\epsilon_{n}:=$ $\exp (2 \pi i / n)$.

Since $Q_{n}=\Pi_{d \mid n} \Phi_{d}$, follows that

$$
n^{n}=\operatorname{Disc}\left(Q_{n}\right)=\Pi_{d, m \mid n, d<m} \operatorname{Res}\left(\Phi_{d}, \Phi_{m}\right) \Pi_{d \mid n} \operatorname{Disc}\left(\Phi_{d}\right) .
$$


The clever calculation of all the factors of the above product was found by Emma Lehmer [Lehm30] in 1930 (in her terminology a simple integer is what is today called a square-free integer) and then reproven with different proofs by several authors [Apo70, [Died40, Dres12]; in particular, the calculation of $\beta_{d, m}$ can be found in an article Dres12] by G. Dresden.

We summarize these results by Lehmer, Diederichsen, Apostol, Dresden with a minor addition (here $\phi(d)$ is the Euler function, i.e. , $\phi(d)=$ $\left.\operatorname{deg} \Phi_{d}\right)$ :

Theorem 2.3. Let $d, m \in \mathbb{Z}, d<m$. Then, if $\beta_{d, m}$ is defined by:

$$
\mathbb{Z} /\left(\left(\Phi_{m}, \Phi_{d}\right) \cap \mathbb{Z}\right)=\mathbb{Z} /\left(\beta_{d, m}\right),
$$

then $\beta_{d, m}$ is \pm 1 unless $d \mid m$ and there exists a prime $p$ such that

$$
m=p^{k} d
$$

in which case $\beta_{d, p^{k} d}=p$.

Moreover, $\operatorname{Res}_{x}\left(\Phi_{d}, \Phi_{p^{k} d}\right)=p^{\phi(d)}$ in the latter case, and 1 otherwise. In particular, $R_{m, d}=0$ unless $d \mid m$ and there exists a prime $p$ such that $m=p^{k} d$, and in this case $R_{d, p^{k} d}$ is a direct sum of finite fields $\mathbb{F}_{p^{\nu}}$ if and only if $d$ is not divisible by $p$.

Moreover, $R_{d, p^{k} d}$ is a field $\mathbb{F}_{p^{\phi(d)}}$ if and only if the class of $p$ generates the group $(\mathbb{Z} / d)^{*}$.

Proof. Only the last assertions need to be proven, since the rest is contained in the cited articles.

Clearly $R_{m, d}=0$ if $\beta_{d, m}$ is \pm 1 , since then $1 \equiv 0$.

If instead $\beta_{d, m}=p, R_{d, p^{k} d}$ is an $\mathbb{F}_{p}=\mathbb{Z} / p$ module, and

$$
R_{d, p^{k} d}=\mathbb{F}_{p}[x]\left(\Phi_{d}, \Phi_{p^{k} d}\right)=\mathbb{F}_{p}[x] /(P),
$$

where $P$ is the G.C.D. of (the reductions $\Psi_{d}, \Psi_{p^{k} d}$ of) $\Phi_{d}, \Phi_{p^{k} d}$ inside $\mathbb{F}_{p}[x]$.

(i) If $d$ is not divisible by $p$, then the polynomial $x^{d}-1$ is square free, and $\mathbb{F}_{p}[x] /\left(x^{d}-1\right)$ is a direct sum of fields. A fortiori $\mathbb{F}_{p}[x] /(P)$ is a direct sum of fields. Indeed we shall show next that $P=\Psi_{d}$.

(ii) The next question is to show that $\Psi_{d} \mid \Psi_{p^{k} d}$, so that $P=\Psi_{d}$ for $d$ not divisible by $p$.

We use the previously cited formula for the cyclotomic polynomial $\Phi_{D}$ when $D=p^{k} d$ and $p$ does not divide $d$ (using the fact that only the terms with $D^{\prime}$ square-free occur):

$$
\begin{gathered}
\Phi_{D}(x)=\Pi_{D^{\prime} \mid D}\left(x^{D / D^{\prime}}-1\right)^{\mu\left(D^{\prime}\right)}= \\
=\prod_{d^{\prime} \mid d}\left(x^{d p^{k} / d^{\prime}}-1\right)^{\mu\left(d^{\prime}\right)} \prod_{d^{\prime} \mid d}\left(x^{d p^{k-1} / d^{\prime}}-1\right)^{-\mu\left(d^{\prime}\right)} .
\end{gathered}
$$

From this we derive, reducing modulo $p$,

$$
\Psi_{D}(x)=\Psi_{p^{k} d}(x)=\Psi_{d}(x)^{\left(p^{k}-p^{k-1}\right)}=\Psi_{d}(x)^{(p-1) p^{k-1}} .
$$


(iii) In the case of $D=p^{k} d$, and where $p \mid d$, we write $d=d_{1} p^{h}$, with $d_{1}$ not divisible by $p$.

The formulae we have just established in (ii) imply

$$
\begin{gathered}
\Psi_{p^{k} d}(x)=\Psi_{p^{k+h} d}(x)=\Psi_{d_{1}}(x)^{\left(p^{k+h-1}(p-1)\right)}, \\
\Psi_{d}(x)=\Psi_{p^{h} d_{1}}(x)=\Psi_{d_{1}}(x)^{\left(p^{h-1}(p-1)\right)},
\end{gathered}
$$

hence again the G.C.D. $P$ equals $\Psi_{d}(x)=\Psi_{d_{1}}(x)^{\left(p^{h-1}(p-1)\right)}$, and $\mathbb{F}_{p}[x] /(P)$ is an algebra with nilpotents.

(iv) Finally, remains to answer the question: when is the reduction $\Psi_{d}$ irreducible? Certainly not in the case where $d \mid(p-1)$ and $\Psi_{d}$ then splits as a product of linear factors (e.g. $\Psi_{4}=\left(x^{2}+1\right)=(x+2)(x+3) \in$ $\left.\mathbb{F}_{5}[x]\right)$.

In general, consider the splitting field of $\left(x^{d}-1\right)$ as an extension of $\mathbb{F}_{p}$. It will be the smallest $\mathbb{F}_{p^{k}}$ which contains the $d$-th roots of 1 , hence $k$ shall be the smallest integer such that $d \mid p^{k}-1 \Leftrightarrow p^{k} \equiv 1(\bmod d)$.

Hence $\Psi_{d}$ irreducible iff the splitting field has degree $k=\phi(d)$, equivalently $p$ is a generator of the group $(\mathbb{Z} / d)^{*}$.

Example 2.4. (i) Consider $R_{4,8}$. It equals the algebra with nilpotents $\mathbb{Z}[x] /\left(x^{2}+1, x^{4}+1\right)=\mathbb{Z}[x] /\left(x^{2}+1,-x^{2}+1\right)=\mathbb{Z}[x] /\left(x^{2}+1,2\right)=\mathbb{F}_{2}[x] /(1+x)^{2}$.

\section{3. $R(n):=\mathbb{Z}\left[C_{n}\right]=\mathbb{Z}[x] /\left(x^{n}-1\right)$-MODULES WHICH ARE TORSION FREE ABELIAN GROUPS.}

In this section $C_{n}$ denotes the cyclic group with $n$ elements, $C_{n} \cong \mathbb{Z} / n$. Hence the group algebra $\mathbb{Z}\left[C_{n}\right]$ is isomorphic to

$$
R(n):=\mathbb{Z}[x] /\left(Q_{n}\right)=\mathbb{Z}[x] /\left(x^{n}-1\right)
$$

and we can apply the results of the previous section.

Let $\Lambda$ be an $R(n)$-module, and assume that $\Lambda$ is a finitely generated torsion free Abelian group.

This hypothesis allows us to view $\Lambda$ as a lattice in the $\mathbb{Q}$-vector space $\Lambda \otimes \mathbb{Q}$, which is therefore also an $R(n)$-module, and an $R(n) \otimes \mathbb{Q}$-module. Since

$R(n) \otimes \mathbb{Q}=\mathbb{Q}[x] /\left(Q_{n}\right)=\mathbb{Q}[x] /\left(\Pi_{d \mid n} \Phi_{d}\right)=\oplus_{d \mid n} \mathbb{Q}[x] /\left(\Phi_{d}\right)=\oplus_{d \mid n} R_{d} \otimes \mathbb{Q}$,

and accordingly (see for instance Lemma 24, page 313 of [Cat15]) we have a splitting

$$
\Lambda \otimes \mathbb{Q}=\oplus_{d \mid n} \Lambda_{d, \mathbb{Q}}
$$

where $\Lambda_{d, \mathbb{Q}}$ is an $R_{d} \otimes \mathbb{Q}$-module, and an $R(n) \otimes \mathbb{Q}$-module via the projection $R(n) \otimes \mathbb{Q} \rightarrow R_{d} \otimes \mathbb{Q}$. 
Definition 3.1. We define $\Lambda_{d}:=\Lambda \cap \Lambda_{d, \mathbb{Q}}$. It is a lattice in $\Lambda_{d, \mathbb{Q}}$, so that we have an exact sequence

$$
0 \rightarrow \oplus_{d \mid n} \Lambda_{d} \rightarrow \Lambda \rightarrow \Lambda^{0} \rightarrow 0,
$$

where $\Lambda^{0}$ is a finite Abelian group.

$\Lambda_{d}$ is an $R_{d}$-module in view of the exact sequence established in section 2 :

$$
0 \rightarrow R(n) \rightarrow \oplus_{d \mid n} R_{d} \rightarrow \oplus_{d_{1}<d_{2}} R_{d_{1}, d_{2}} \rightarrow 0
$$

which shows that $R(n)$ acts on $\Lambda_{d, \mathbb{Q}}$ via the homomorphism $R(n) \rightarrow R_{d}$.

We can make the geometry of the above exact sequences more transparent if we introduce the associated real tori.

Definition 3.2. Given an $R(n)$-module $\Lambda$, which is a lattice, i.e., a free Abelian group of finite rank, we define the associated tori as:

(1) $A:=(\Lambda \otimes \mathbb{R}) / \Lambda$, and since

(2) $\Lambda \otimes \mathbb{R}=\oplus_{d \mid n} \Lambda_{d, \mathbb{R}}$, we define

(3) $A_{d}:=\Lambda_{d, \mathbb{R}} / \Lambda_{d}$, hence

(4) we have an exact sequence

$$
0 \rightarrow \Lambda^{0} \rightarrow \oplus_{d \mid n} A_{d} \rightarrow A \rightarrow 0
$$

identifying the cokernel $\Lambda^{0}$ as a finite subgroup of the product torus $A^{\prime}:=\oplus_{d \mid n} A_{d}$, isogenous to $A$,

(5) $R^{\prime}(n):=\oplus_{d \mid n} R_{d}$ acts on $A^{\prime}$, and we set

(6) $R^{0}(n):=\oplus_{d_{1}<d_{2}} R_{d_{1}, d_{2}}$, so that

(7) we have the exact sequence $0 \rightarrow R(n) \rightarrow R^{\prime}(n) \rightarrow R^{0}(n) \rightarrow 0$.

Proposition 3.1. The datum of an $R(n)$ module which is a lattice, i.e., a free Abelian group of finite rank, is equivalent to the datum of torsion free $R_{d^{-}}$-modules $\Lambda_{d}$ of finite rank, and of a finite subgroup $\Lambda^{0} \subset A^{\prime}:=\oplus_{d \mid n} A_{d}$, where $A_{d}:=\Lambda_{d, \mathbb{R}} / \Lambda_{d}$, such that:

(A) $\Lambda^{0}$ is stable for the subring $R(n)$, which is equivalent to the requirement: $x \Lambda^{0}=\Lambda^{0}\left(\Leftrightarrow x \Lambda^{0} \subset \Lambda^{0}\right)$

(B) $\Lambda^{0} \cap A_{d}=0 \forall d \mid n$.

Properties (A) and (B) imply:

(C) $\frac{Q_{n}}{\Phi_{d}}(\lambda) \in \Lambda_{d}, \forall d \mid n, \forall \lambda \in \Lambda$;

hence, writing an element of $\Lambda^{0}$ as $\left(\lambda_{d}\right)_{d \mid n}$, we have

$$
\lambda_{d} \in\left(\frac{\Phi_{d}}{Q_{n}} \Lambda_{d}\right) / \Lambda_{d} \cong \Lambda_{d} /\left(\frac{Q_{n}}{\Phi_{d}}\right)
$$

and it follows that

(D) The number of such finite subgroups $\Lambda^{0}$ is finite.

Proof. Clearly, $\Lambda$ is determined by the subgroup $\Lambda^{0} \subset A^{\prime}$, and the property that $\Lambda$ is stable for the subring $R(n)$ is equivalent to property (A) that $R(n)$ stabilizes $\Lambda^{0}$. 
Property (B) ensures that $\Lambda \cap \Lambda_{d, \mathbb{R}}=\Lambda_{d}$.

Property (C) follows right away since $\frac{Q_{n}}{\Phi_{d}}(\lambda) \in \Lambda$, but its components in $\Lambda_{d^{\prime}, \mathbb{Q}}$ are $=0$ for $d^{\prime} \neq d$, hence this element lies in $\Lambda_{d}$.

Property (D) follows since $\Lambda^{0} \subset \oplus_{d \mid n}\left(\frac{\Phi_{d}}{Q_{n}} \Lambda_{d}\right) / \Lambda_{d}$, which is finite group since $\left(\frac{\Phi_{d}}{Q_{n}} \Lambda_{d}\right) / \Lambda_{d} \cong \Lambda_{d} /\left(\frac{Q_{n}}{\Phi_{d}}\right)$ is a finite module over the finite ring $\mathbb{Z}[x] /\left(\Phi_{d}, \frac{Q_{n}}{\Phi_{d}}\right)$ that we have been describing in the previous section.

The previous proposition is particularly useful in the case where the Dedekind ring $R_{d}$ is a PID (Principal Ideal Domain), because then every torsion free $R_{d}$-module is free.

In fact, more generally (see [Miln71]) every torsion free module over a Dedekind domain $R$ is the direct sum of a free module with an ideal $I$, hence $R$ is a PID iff every torsion free module is free.

However, how does the above description of $R(n)$-modules apply to the free module $R(n)$ ?

The answer is related to finding the inverse map in the Chinese remainder theorem, of which theorem 1.1 is a generalization.

Proposition 3.2. Consider the following module-homomorphism

$$
j: R^{\prime}(n)=\oplus_{d \mid n} R_{d} \rightarrow R(n),
$$

such that $\left.j\right|_{R_{d}}$ is induced by multiplication with $Q_{n} / \Phi_{d}$ (recall that $\left.R_{d}=\mathbb{Z}[x] /\left(\Phi_{d}\right)\right)$.

1) Composing with the natural inclusion $i: R(n) \rightarrow R^{\prime}(n)$ we obtain an injective map:

$$
\psi: R^{\prime}(n)=\oplus_{d \mid n} R_{d} \rightarrow R^{\prime}(n)=\oplus_{d \mid n} R_{d},
$$

which is of diagonal form.

2) We have $j\left(R_{d}\right)=R(n) \cap\left(R_{d} \otimes \mathbb{Q}\right) \subset R(n) \otimes \mathbb{Q}$.

Proof. 1) means that $\psi\left(R_{d}\right) \subset R_{d}$, which follows since $Q_{n} / \Phi_{d} \equiv 0 \in R_{d^{\prime}}$ for $d^{\prime} \neq d$.

2) follows since $R(n) \cap\left(R_{d} \otimes \mathbb{Q}\right)$ is the kernel of $R(n) \rightarrow \oplus_{d^{\prime} \mid n, d^{\prime} \neq d} R_{d^{\prime}}$, hence it is an $R(n)$-module, hence an ideal in $R(n)$ : and it must be the ideal generated by $\prod_{d^{\prime} \mid n, d^{\prime} \neq d} \Phi_{d^{\prime}}=Q_{n} / \Phi_{d}$.

We also notice that $\psi \otimes \mathbb{Q}$ is an isomorphism, and that we have a surjection

$$
\operatorname{Coker}(\psi) \rightarrow \operatorname{Coker}(i)
$$

in view of the injective maps

$$
j: R^{\prime}(n) \rightarrow R(n), i: R(n) \rightarrow R^{\prime}(n)
$$

whose composition is $\psi$. 
$\operatorname{Coker}(\psi)$ is essentially the double of $\operatorname{Coker}(i)$, since

$\operatorname{Coker}(\psi)=\oplus_{d \mid n} R_{d} /\left(Q_{n} / \Phi_{d}\right)=\oplus_{d \mid n} \mathbb{Z}[x] /\left(\Phi_{d}, Q_{n} / \Phi_{d}\right)=\oplus_{d \mid n} \mathbb{Z}[x] /\left(\Phi_{d}, \Pi_{d^{\prime} \neq d} \Phi_{d}^{\prime}\right)$.

Now,

$$
R_{d} /\left(\Pi_{d^{\prime} \neq d} \Phi_{d}^{\prime}\right)
$$

is by Corollary 1.2 isomorphic to the finite ring

$$
\oplus_{d^{\prime} \mid n, d^{\prime} \neq d} R_{d, d^{\prime}}
$$

Therefore, we have the surjection:

$$
\operatorname{Coker}(\psi)=\oplus_{d \mid n} R_{d} /\left(\Pi_{d^{\prime} \neq d} \Phi_{d}^{\prime}\right) \rightarrow \operatorname{Coker}(i)=\oplus_{d, d^{\prime} \mid n, d<d^{\prime}} R_{d, d^{\prime}}
$$

and an exact sequence

$$
0 \rightarrow \operatorname{Coker}(j) \rightarrow \operatorname{Coker}(\psi) \rightarrow \operatorname{Coker}(i) \rightarrow 0,
$$

and this shows that we have an isomorphism

$$
\operatorname{Coker}(j) \cong \operatorname{Coker}(i)=\oplus_{d, d^{\prime} \mid n, d<d^{\prime}} R_{d, d^{\prime}} .
$$

We can summarize everything in the following

Proposition 3.3. We have a sequence of inclusions:

$$
0 \rightarrow M^{\prime}:=\oplus_{d \mid n}\left(Q_{n} / \Phi_{d}\right) R_{d} \rightarrow R(n) \rightarrow R^{\prime}:=\oplus_{d \mid n} R_{d},
$$

such that

$$
R^{0}(n):=R(n) / M^{\prime} \subset R^{\prime} / M^{\prime}=\oplus_{d, d^{\prime} \mid n, d \neq d^{\prime}} R_{d, d^{\prime}}
$$

is identified as the submodule of $R^{\prime} / M^{\prime}$,

$$
R^{0}(n)=\left\{\left(a_{d, d^{\prime}}\right) \mid\left(a_{d, d^{\prime}}\right)=\left(a_{d^{\prime}, d}\right)\right\} .
$$

Proof. There remains only to prove the last assertion, which follows immediately from the observation that $R(n)$ is the kernel of the map to $\oplus_{d, d^{\prime} \mid n, d<d^{\prime}} R_{d, d^{\prime}}$, given by taking differences $b_{d}-b_{d^{\prime}}$ of the image of $b \in R(n)$ to $R_{d}$.

Remark 3.3. Let us go back to example [3.3, where the divisors of $n=6$ are $1,2,3,6$ and the only nonzero $R_{d, d^{\prime}}$ 's are:

- $R_{1,2}=\mathbb{Z} / 2$ acted trivially by $x$, since $x \equiv 1$,

- $R_{1,3}=\mathbb{Z} / 3$ acted trivially by $x$, since $x \equiv 1$,

- $R_{2,6}=\mathbb{Z} / 3$, where $x$ acts multiplying by -1 , since $x+1 \equiv 0$,

- $R_{3,6}=\mathbb{F}_{4}$, with $\mathbb{Z} / 2$ basis $1, x$ and with $x^{2} \equiv 1+x$.

We can now apply the method of proposition 3.1 to construct many $\Lambda^{0} \subset R^{\prime} / M^{\prime}=\oplus_{d, d^{\prime} \mid n, d \neq d^{\prime}} R_{d, d^{\prime}}$.

For instance, we may take

$$
\Lambda^{0}=\left\{\left(a_{d, d^{\prime}}\right) \mid a_{1,2}=a_{2,1}=0, a_{1,3}=a_{3,1}, a_{2,6}=a_{6,2}, a_{3,6}=a_{6,3}=0\right\},
$$

and we get a module different from $R(6)$. 


\section{Fully RAmified CyClic COVERINGS of the PROJective Line AND ASSOCIATED Hodge STRUCTURES}

Let $f: C \rightarrow \mathbb{P}^{1}$ be a cyclic covering with Galois group

$$
\mu_{n}=\left\{\zeta \in \mathbb{C} \mid \zeta^{n}=1\right\} \cong \mathbb{Z} / n,
$$

branched on $k+2$ points $P_{0}=0, P_{1}=1, P_{2}, \ldots, P_{k}, \infty$, and let us assume that $C$ is the normalization of the affine curve of equation

$$
y^{n}=x^{m_{0}}(x-1)^{m_{1}}\left(x-t_{2}\right)^{m_{2}} \ldots\left(x-t_{k}\right)^{m_{k}},
$$

where $P_{i}=\left\{x=t_{i}\right\}$ and where without loss of generality we may assume $1 \leq m_{j}<n$.

The local monodromy of the covering around the point $P_{i}$ sends the standard local generator to the element $m_{i} \in \mathbb{Z} / n$, hence the inertia group of $P_{i}$ is cyclic of order $r_{i}=\frac{n}{\text { G.C.D. }\left(n, m_{i}\right)}$.

Definition 4.1. One says that a Galois covering is fully ramified if there is a branch point whose inverse image consists of only one point. In the case of curves, this implies that the Galois covering is cyclic with group $\mathbb{Z} / n$, and there is a branch point $P_{i}$ with $r_{i}=n$.

In the following, we shall make the assumption that $f: C \rightarrow \mathbb{P}^{1}$ is fully ramified, and without loss of generality we may assume that $m_{0}=1$. Our goal, in this section, is to describe $H_{1}(C, \mathbb{Z})$ as an $R(n)=\mathbb{Z}\left[\mu_{n}\right]$ module.

Recall that the fundamental group $\pi_{1}(C)$ is the kernel of the following exact sequence (see for instance [Cat08, pages 101-104):

$$
1 \rightarrow \pi_{1}(C) \rightarrow \mathcal{T}:=T\left(0 ; r_{0}, r_{1}, \ldots, r_{k}, r_{\infty}\right) \rightarrow \mathbb{Z} / n \rightarrow 0,
$$

where the polygonal orbifold group $\mathcal{T}:=T\left(0 ; r_{0}, r_{1}, \ldots, r_{k}, r_{\infty}\right)$ has generators

and relations

$$
\gamma_{0}, \gamma_{1}, \ldots, \gamma_{k}, \gamma_{\infty}
$$

$$
\gamma_{i}^{r_{i}}=1, \forall i, \gamma_{0} \cdot \gamma_{1} \cdots \gamma_{k} \cdot \gamma_{\infty}=1 .
$$

Breaking the symmetry, we shall see $\gamma_{0}, \gamma_{1}, \ldots \gamma_{k}$ as generators for $\mathcal{T}$, and $\gamma_{0}^{n}=1, \gamma_{1}^{r_{1}}=1, \ldots, \gamma_{k}^{r_{k}}=1,\left(\gamma_{0} \cdot \gamma_{1} \cdots \gamma_{k}\right)^{r_{\infty}}=1$ as relations.

One sees then immediately that $\pi_{1}(C)$ is generated by $1+n(k)$ elements, and, since $\gamma_{0}, \gamma_{0}^{2}, \ldots, \gamma_{0}^{n-1}$ is a Schreier system, we can choose, by the Reidemeister Schreier method ([MKS], theorem 2.7, page 89 and following) the following generators:

$$
\gamma_{0}^{n}, \delta_{i, j}:=\gamma_{0}^{i} \gamma_{j} \gamma_{0}^{-m_{j}-i}, i=0, \ldots, n-1, j=1, \ldots, k .
$$

These generators are nice because the Galois group $\mathbb{Z} / n$ acts on $\pi_{1}(C)$ by conjugation of a lift of $i \in \mathbb{Z} / n$, hence by conjugation by $\gamma_{0}^{i}$. Hence these elements are permuted by the Galois group. 
It is obvious that we can forget about the first generator $\gamma_{0}^{n}$ in view of the relation $\gamma_{0}^{n}=1$.

The Hurwitz formula calculates the genus $g$ of the curve $C$ as follows:

$$
2 g-2=n\left(-2+\sum_{i=0, \ldots, k, \infty} \frac{\left(r_{i}-1\right)}{r_{i}}\right)=\sum_{i=0, \ldots, k, \infty}\left(n-\frac{n}{r_{i}}\right)-2 n,
$$

hence

$$
2 g=\sum_{i=1, \ldots, k, \infty}\left(n-\frac{n}{r_{i}}\right)-n+1=\sum_{i=1, \ldots, k}\left(n-\frac{n}{r_{i}}\right)+1-\frac{n}{r_{\infty}}
$$

We shall further reduce the number of generators using the other relations, until we reach $2 g$ generators: the classes of these in $H_{1}(C, \mathbb{Z})=$ $\pi_{1}(C)^{a b}$ will then give a basis for the first homology group.

Indeed, we can rewrite:

$$
1=\gamma_{j}^{r_{j}}=\gamma_{0}^{i} \gamma_{j}^{r_{j}} \gamma_{0}^{-i}=\delta_{i, j} \delta_{i+m_{j}, j} \delta_{i+2 m_{j}, j} \ldots \delta_{i+\left(r_{j}-1\right) m_{j}, j} .
$$

Case $r_{\infty}=n$ : We can eliminate in this way, since $\left(m_{j}\right) \subset \mathbb{Z} / n$ equals $\left(n / r_{j}\right) \subset \mathbb{Z} / n, \sum_{i=1, \ldots, k} \frac{n}{r_{i}}$ generators, and we obtain the right number of generators, $\sum_{i=1, \ldots, k}\left(n-\frac{n}{r_{i}}\right)=2 g$ generators. The rewriting of the relations coming from $\gamma_{\infty}^{r_{\infty}}=1$ yields the standard relation for $\pi_{1}(C)$.

Definition 4.2. Define $D_{i, j}$ as the class of $\delta_{i, j}$ inside $H_{1}(C, \mathbb{Z})$, for $i=0, \ldots, n-1$, and $j=1, \ldots k$.

Then the previous relation rewrites as

$$
\sum_{h=0, \ldots, r_{j}-1} D_{i+h n / r_{j}, j}=0
$$

We have therefore proven

Theorem 4.3. Assume that we have a cyclic covering $f: C \rightarrow \mathbb{P}^{1}$ with group $\mathbb{Z} / n$ and with two points of full ramification.

Then, if the order of the inertia groups are $n, r_{1}, \ldots, r_{k}, n$, the $\mathbb{Z}[x]$ module $H_{1}(C, \mathbb{Z})$ is a direct sum of cyclic modules,

$$
H_{1}(C, \mathbb{Z})=\oplus_{1}^{k}\left(\mathbb{Z}[x] /\left(1+x^{n / r_{j}}+x^{2 n / r_{j}}+\cdots+x^{\left(r_{j}-1\right) n / r_{j}}\right) .\right.
$$

Example 4.4. This example is borrowed from the modular description of the Cartwright-Steger surface, which was first explained to me by Domingo Toledo.

Assume that we have $n=12$, and $m_{0}=7, m_{1}=m_{2}=m_{3}=2, m_{\infty}=$ 11 , hence ramification indices $(12,6,6,6,12)$.

Then $H_{1}(C, \mathbb{Z}) \cong \oplus_{1}^{6} \mathbb{Z}[x] /\left(x^{10}+x^{8}+\cdots+1\right)$.

Since $x^{10}+x^{8}+\cdots+1=\left(x^{12}-1\right) /\left(x^{2}-1\right)=\Phi_{3} \Phi_{4} \Phi_{6} \Phi_{12}$, we see that, by the previous results, setting

$$
M:=\mathbb{Z}[x] /\left(x^{10}+x^{8}+\cdots+1\right),
$$

we have $H_{1}(C, \mathbb{Z}) \cong 3 M$, and 
$M \supset \Phi_{4} \Phi_{6} \Phi_{12} M \oplus \Phi_{3} \Phi_{6} \Phi_{12} M \oplus \Phi_{3} \Phi_{4} \Phi_{12} M \oplus \Phi_{3} \Phi_{4} \Phi_{6} M \cong R_{3} \oplus R_{4} \oplus R_{6} \oplus R_{12}$.

An easy calculation shows that the $i$-th dimensional eigenspace $V_{j}:=$ $H^{0}\left(\Omega_{C}^{1}\right)^{j}$ has dimension $v(j)$ with:

$v(j)=0$, for $j=0,5,6, v(j)=2$, for $j=1,2,8,9, v(j)=1$, for $j=3,4,7,10,11$.

Now, we consider the cohomology $H^{1}(C, \mathbb{Z})$, again as a $\mathbb{Z}[x]$-module.

Then $H^{1}(C, \mathbb{Z})=3 \operatorname{Hom}(M, \mathbb{Z})$, and by the exact sequence

$$
0 \rightarrow M \rightarrow \sum_{d=3,4,6,12} M / \Phi_{d} M=M \otimes_{\mathbb{Z}[x]} R_{d}=R_{d} \rightarrow \text { Coker } \rightarrow 0
$$

we find that $\operatorname{Hom}(M, \mathbb{Z})$ contains

$$
\sum_{d=3,4,6,12} \operatorname{Hom}\left(M / \Phi_{d} M, \mathbb{Z}\right)=\sum_{d=3,4,6,12} \operatorname{Hom}\left(R_{d}, \mathbb{Z}\right) \cong \sum_{d=3,4,6,12} R_{d}
$$

where the duality $R_{d} \times R_{d} \rightarrow \mathbb{Z}$ is given by the product followed by the trace map.

Remark 4.5. Theorem 4.3 works if there are two points of full ramification; if there is exactly one, we are in the situation of

Case 2: $r_{1}, r_{2}, \ldots r_{k}, r_{\infty}<n$.

Here we can use the relation

$$
\left(\gamma_{0} \cdot \gamma_{1} \cdots \gamma_{k}\right)^{r_{\infty}}=1
$$

to rewrite

$$
\begin{gathered}
1=\gamma_{0}^{i}\left(\gamma_{0} \cdot \gamma_{1} \cdots \gamma_{k}\right)^{r_{\infty}} \gamma_{0}^{-i}= \\
=\delta_{i+1,1} \cdot \delta_{i+1+m_{1}, 2} \ldots \delta_{i+1+m_{1}+\cdots+m_{k-1}, k} \delta_{i+1-m_{\infty}, 1} \ldots \delta_{i-m_{k}, k}=1 .
\end{gathered}
$$

These, since $\mathbb{Z} /\left(m_{\infty}\right)=\mathbb{Z} /\left(n / r_{\infty}\right)$, are $n / r_{\infty}$ relations, as expected.

Passing to the Abelianization of $\pi_{1}(C)$, we get the extra relations:

$$
\sum_{h=0, \ldots, r_{\infty}-1} D_{i+1+h n / r_{\infty}, 1}+D_{i+1+m_{1}+h n / r_{\infty}, 2}+\cdots+D_{i-m_{k}+h n / r_{\infty}, k}=0 .
$$

This case should be easier to treat than the general one with no points of full ramification.

\section{Structure Theorem for Bagnera-De Franchis MANIFOLDS}

The goal of this section is to give a complete structure theorem for Bagnera-De Franchis Manifolds, leaving aside the question of projectivity, which was treated in [CD17] (in the appendix it was shown that each BdF Manifold deforms to a projective one).

Set in this section $G=\mathbb{Z} / n$ and consider a Bagnera-De Franchis Manifold $X=A / G$, where $A$ is a complex torus $A=V / \Lambda$ of dimension $g$. Here we use the letter $A$ even if we have a torus, and not necessarily 
an Abelian variety, just in order to have a similar notation to Cat15] (where the letter $T$ was used to denote some torsion subgroup).

Holomorphic maps $F: A \rightarrow A^{\prime}$ of complex tori are affine maps, since their derivatives in the flat uniformizing parameters are constant: hence such holomorphic maps

$$
F: A=V / \Lambda \rightarrow A^{\prime}=V^{\prime} / \Lambda^{\prime}
$$

can be represented as

$$
F(v)=\alpha(v)+b\left(\bmod \Lambda^{\prime}\right), \alpha: V \rightarrow V^{\prime}, \alpha(\Lambda) \subset \Lambda^{\prime}, b \in V^{\prime} .
$$

$\alpha$ is a linear map of vector spaces induced by $\left.\alpha\right|_{\Lambda}$, which we still denote by $\alpha$ : indeed, any $\mathbb{Z}$-linear map $\alpha:(\Lambda) \rightarrow \Lambda^{\prime}$ induces a complex linear map

$$
\alpha \otimes \mathbb{C}: \Lambda \otimes \mathbb{C}=V \oplus \bar{V} \rightarrow \Lambda^{\prime} \otimes \mathbb{C}=V^{\prime} \oplus \overline{V^{\prime}},
$$

and $\alpha$ induces a homomorphism of complex tori if and only if

$$
(\alpha \otimes \mathbb{C})(V) \subset V^{\prime},
$$

i.e. $\alpha \otimes \mathbb{C}$ is a homomorphism of Hodge structures.

We take now a generator $\gamma \in G$, and write $\gamma(v)=\alpha(v)+b$ (here $A^{\prime}=A$ ).

Then we have a decomposition $V=\oplus_{\zeta \in \mu_{n}} V_{\zeta}$, where $V_{\zeta}$ is the eigenspace for the complex linear map $\alpha$ corresponding to the eigenvalue $\zeta$.

The condition that $\gamma$ has no fixed point means that there is no solution of the equation

$$
\alpha(v)+b \equiv v(\bmod \Lambda) \Leftrightarrow(\alpha-I d)(v)+b \in \Lambda .
$$

Writing $V=V_{1} \oplus V_{2}, V_{2}:=\oplus_{\zeta \in \mu_{n}, \zeta \neq 1} V_{\zeta}$, we have that $(\alpha-I d)$ is invertible on $V_{2}$, hence after a change of the origin we may assume that $b \in V_{1}$, and that

$$
\gamma\left(v_{1}, v_{2}\right)=\left(v_{1}+b_{1}, \alpha_{2}\left(v_{2}\right)\right)
$$

The condition that $G$ operates freely on $A$ amounts to:

$$
(* *) \exists\left(\lambda_{1}, \lambda_{2}\right) \in \Lambda \text { such that } h b_{1}=\lambda_{1} \Leftrightarrow n \mid h .
$$

$\alpha$ makes $\Lambda$ an $R(n)$ module, hence we have (compare the notation introduced just before Proposition [3.1) the decomposition $\Lambda \otimes \mathbb{Q}=$ $\oplus_{d \mid n} \Lambda_{d, \mathbb{Q}}$, and setting

$$
\Lambda_{d}:=\Lambda \cap \Lambda_{d, \mathbb{Q}}, \Lambda^{0}:=\Lambda / \oplus_{d \mid n} \Lambda_{d}, A_{d}:=\Lambda_{d, \mathbb{R}} / \Lambda_{d}
$$

we have an exact sequence

$$
0 \rightarrow \Lambda^{0} \rightarrow A^{\prime}:=\oplus_{d \mid n} A_{d} \rightarrow A \rightarrow 0 .
$$

Theorem 5.1. A Bagnera-De Franchis Manifold with group $G=\mathbb{Z} / n$ is completely determined by the following data:

(1) the datum of torsion free $R_{d}$-modules $\Lambda_{d}$ of finite rank, for all $d \mid n$, such that $\Lambda_{1} \neq 0$ and with $\Lambda_{1}, \Lambda_{2}$ of even rank; 
(2) the datum of a finite subgroup $\Lambda^{0} \subset A^{\prime}:=\oplus_{d \mid n} A_{d}$, where $A_{d}:=$ $\Lambda_{d, \mathbb{R}} / \Lambda_{d}$

(3) an element $\beta_{1} \in A_{1}$ generating a subgroup $\left\langle\beta_{1}\right\rangle$ of order exactly $n$, such that:

(4) (A) $\Lambda^{0}$ is stable for multiplication by the element $x$ of the subring $R(n) \subset R^{\prime}(n):=\oplus_{d \mid n} R_{d}$, and

(5) (B) $\Lambda^{0} \cap A_{d}=0 \forall d \mid n$,

(6) $(C)$ the projection of $\Lambda^{0}$ into $A_{1}$ intersects the subgroup $\left\langle\beta_{1}\right\rangle$ only in 0 ;

(7) the datum of a complex structure on each $\Lambda_{d} \otimes \mathbb{C}$, i.e., a Hodge decomposition

$$
\Lambda_{d} \otimes \mathbb{C}=V(d) \oplus \overline{V(d)},
$$

which allows to decompose $V(d)=\oplus_{j<d,(j, d)=1} V_{j}$ as a direct sum of eigenspaces for the action $\alpha$ of $x$.

(8) The properties (A) and (B) imply that $\Lambda^{0} \subset \oplus_{d \mid n}\left(\frac{\Phi_{d}}{Q_{n}} \Lambda_{d}\right) / \Lambda_{d}$, hence, in particular, the number of such subgroups $\Lambda^{0}$ is finite.

Proof. According to Proposition 3.1 the data (1) and (2), provided that (4) and (5) hold, determine a lattice $\Lambda$ which is an $R(n)$-module.

The conditions in (1) that $\Lambda_{1}, \Lambda_{2}$ have even rank are necessary for the existence of a complex structure on $\Lambda_{d} \otimes \mathbb{C}$ for $d=1,2$.

We can then choose $V_{1}=V(1)$ to give a Hodge structure on $\Lambda_{1} \otimes \mathbb{C}$ and, if $n$ is even, $V(2)=V_{n / 2}$ to give a complex structure on $\Lambda_{2} \otimes \mathbb{C}$. Whereas, for $d \geq 3, \Lambda_{d} \otimes \mathbb{C}$ splits as a direct sum of eigenspaces $W_{j}$, corresponding to the eigenvalues $\epsilon_{n}^{j n / d}$, for $j<d,(j, d)=1$.

These eigenvalues come in conjugate pairs, hence it suffices to consider $W_{j} \oplus W_{n-j}$ and choose $V_{j}$, for $j<d / 2$, to be any subspace of $W_{j}$, letting then $V_{n-j} \subset W_{n-j}$ be a subspace such that $W_{j}=V_{j} \oplus \overline{V_{n-j}}$.

We are done, since $W_{n-j}=\overline{W_{j}}$.

Finally, we take the transformation $\gamma$ whose linear part is the linear map $\alpha$ corresponding to multiplication by $x$, and whose translation part is $\beta_{1} \in A_{1}$. The condition that any power of $\gamma$ with exponent $h<n$ has no fixed points means (see $(* *))$ that the equation $\left(\gamma^{h}(v)-v\right) \in \Lambda$ has no solutions $v \in V$. Let $\beta_{1}$ be the class of $b \in V_{1}$ : then this equation is equal to

$$
h b+\left(\alpha^{h}-I d\right)(v) \in \Lambda .
$$

Since the image of $\left(\alpha^{h}-I d\right)$ equals to $V_{2}$, this means that $h b$ does not belong to the projection of $\Lambda$ into $V_{1}$. This is equivalent to requiring that $h \beta_{1}$ does not belong to the projection of $\Lambda^{0}$ into $A_{1}$.

Property (8) was already shown in Proposition 3.1 .

Remark 5.2. (I) To relate the formulation given here with the content of Proposition 16 of Cat15, it suffices to define $\Lambda_{2}:=\Lambda \cap V_{2}$, and $T:=\Lambda /\left(\Lambda_{1}+\Lambda_{2}\right)$. Then $T$ is isomorphic to the image of $\Lambda^{0}$ inside $A_{1}$, 
which was called $T_{1}$ in loc. cit. . Hence one requires $T_{1}$ and $\left\langle\beta_{1}\right\rangle$ to intersect only in 0 , and clearly $X=A / G=\left(A_{1} \times A_{2}\right) / G \times T$.

(II) On page 313, eight lines from the bottom of [Cat15] there is a 'lapsus calami', asserting the splitting $R(m)=\oplus_{d \mid m} R_{d}$ without tensoring with $\mathbb{Q}$. However, fortunately, this wrong assertion is not used at all in Cat15.

\section{The intersection PRoduct For the Homology of FUlly RAMIFIED CYCLIC COVERINGS OF THE LINE}

In this section we use the presentation of the fundamental group of a cyclic covering $f: C \rightarrow \mathbb{P}^{1}$ as described in section 4 , and shall determine the intersection product map $H_{1}(C, \mathbb{Z}) \times H_{1}(C, \mathbb{Z}) \rightarrow \mathbb{Z}$ dual to the cup product for the first homology group.

We shall make the assumption that the ramification indices $r_{0}=r_{\infty}=$ $n$.

Then we have a set $\mathcal{G}_{1}$ generators for $\pi_{1}(C)$, consisting of the $n \cdot k$ elements:

$$
\delta_{i, j}:=\gamma_{0}^{i} \gamma_{j} \gamma_{0}^{-m_{j}-i}, i=0, \ldots, n-1, j=1, \ldots, k .
$$

As we saw, the $\sum_{i=1, \ldots, k} \frac{n}{r_{i}}$ relations:

$$
1=\delta_{i, j} \delta_{i+m_{j}, j} \delta_{i+2 m_{j}, j} \ldots \delta_{i+\left(r_{j}-1\right) m_{j}, j},
$$

allow to eliminate $\sum_{i=1, \ldots, k} \frac{n}{r_{i}}$ of these generators, and we obtain a set $\mathcal{G}_{2}$ of generators, with the right number $\sum_{i=1, \ldots, k}\left(n-\frac{n}{r_{i}}\right)=2 g$ of elements. It is convenient to eliminate the generators $\delta_{i, j}, i=0, \ldots, \frac{n}{r_{i}}-1$, and each of them is then equal to the product

$$
\left[\delta_{i+m_{j}, j} \delta_{i+2 m_{j}, j} \ldots \delta_{i+\left(r_{j}-1\right) m_{j}, j}\right]^{-1}=\delta_{i+\left(r_{j}-1\right) m_{j}, j}^{-1} \ldots \delta_{i+2 m_{j}, j}^{-1} \delta_{i+m_{j}, j}^{-1} .
$$

Observe that this is the product of exactly $\left(r_{j}-1\right)$ inverses of elements in the set $\mathcal{G}_{2}$ of $2 g$ generators.

We consider now the relation which is the Reidemeister-Schreier rewriting of $\gamma_{\infty}^{n}=1$.

We have:

$$
\begin{gathered}
1=\left(\gamma_{0} \gamma_{1} \gamma_{2} \ldots \gamma_{k}\right)^{n}= \\
\left(\gamma_{0} \gamma_{1} \gamma_{0}^{-1}\right)\left(\gamma_{0} \gamma_{2} \gamma_{0}^{-1}\right) \ldots\left(\gamma_{0} \gamma_{k} \gamma_{0}^{-1}\right) . \\
\left(\gamma_{0}^{2} \gamma_{1} \gamma_{0}^{-2}\right)\left(\gamma_{0}^{2} \gamma_{2} \gamma_{0}^{-2}\right) \ldots\left(\gamma_{0}^{2} \gamma_{k} \gamma_{0}^{-2}\right) . \\
\ldots \\
\left(\gamma_{0}^{n-1} \gamma_{1} \gamma_{0}^{-(n-1)}\right)\left(\gamma_{0}^{n-1} \gamma_{2} \gamma_{0}^{-(n-1)}\right) \ldots\left(\gamma_{0}^{n-1} \gamma_{k} \gamma_{0}^{-(n-1)}\right) . \\
\gamma_{1} \gamma_{2} \ldots \gamma_{k} .
\end{gathered}
$$

We are then ready to rewrite in terms of the 'big' set $\mathcal{G}_{1}$ of generators:

$$
1=\delta_{1,1} \ldots \delta_{1, k} \delta_{2,1} \ldots \delta_{2, k} \ldots \delta_{n-1,1} \ldots \delta_{n-1, k} \delta_{0,1} \ldots \delta_{0, k} .
$$

This relation is then equal to the product of the $n \cdot k$ original generators. 
Now, we replace, as indicated above, the generators $\delta_{i, j}, i=0, \ldots \frac{n}{r_{i}}$ by the respective products of inverses of the final set $\mathcal{G}_{2}$ of $2 g$ generators. We are then left with a relation where do occur exactly the $2 g$ generators in $\mathcal{G}_{2}\left(2 g=n k-\sum_{i=1, \ldots, k} \frac{n}{r_{i}}\right)$ with exponent equal to +1 , and exactly

$$
\sum_{i=1, \ldots, k} \frac{n}{r_{i}}\left(r_{i}-1\right)=\sum_{i=1, \ldots, k} n-\frac{n}{r_{i}}=2 g
$$

different generators with exponent equal to -1 .

Hence the relation, even if not in the standard form, depicts a 2dimensional manifold obtained attaching a 2-disk to a bouquet of $2 g$ circles.

The recipe for the intersection product is then given in the following

Proposition 6.1. Let $\pi_{g}$ be a group with $2 g$ generators, $a_{1}, \ldots a_{2 g}$, and with only one relation $W\left(a_{1}, \ldots, a_{2 g}\right)=1$, where the word $W\left(a_{1}, \ldots, a_{2 g}\right)$ consists of exactly $4 g$ letters, of which $2 g$ are exactly the letters $a_{1}, \ldots a_{2 g}$, and $2 g$ are exactly the inverses of the letters $a_{1}, \ldots a_{2 g}$.

Then $\pi_{g}$ is the fundamental group of a closed Riemann surface $C$ of dimension $g$, and the intersection product on $H_{1}(C, \mathbb{Z})=\pi_{g}^{a b}$ is determined as follows, considering the word as giving a cyclical order in the set (of cardinality $4 \mathrm{~g}$ ) consisting of the generators $a_{i}$ and of their inverses:

(1) $a_{i} \cdot a_{j}=0$ if, removing $a_{i}$ and $a_{i}^{-1}, a_{j}$ and $a_{j}^{-1}$ lie in the same of the two remaining intervals;

(2) $a_{i} \cdot a_{j}=1$ if, removing $a_{i}$ and $a_{i}^{-1}, a_{j}$ lies in the interval going from $a_{i}$ to $a_{i}^{-1}$, and $a_{j}^{-1}$ lies in the other;

(3) $a_{i} \cdot a_{j}=-1$ if, removing $a_{i}$ and $a_{i}^{-1}, a_{j}^{-1}$ lies in the interval going from $a_{i}$ to $a_{i}^{-1}$, and $a_{j}$ lies in the other.

Proof. Consider a bouquet of $2 g$ circles meeting in one point $P$, and corresponding to the generators $a_{1}, \ldots a_{2 g}$.

We attach a 2 -disk whose boundary is the word $W\left(a_{1}, \ldots, a_{2 g}\right)$. Since in the word each generator $a_{i}$ and its inverse $a_{i}^{-1}$ appear exactly once, the space $C$ that we obtain is smooth outside of $P$. At $P$ however we have $4 g$ segments coming in, and we fill in $4 g$ angles, hence $C$ is a topological manifold. It is also oriented since $H_{2}(C, \mathbb{Z})=\mathbb{Z}$.

The two incoming segments corresponding to $a_{i}$ and $a_{i}^{-1}$ (who are oriented) locally separate $C$ in a neighbourhood of $P$.

In case (1), two incoming segments corresponding to $a_{j}$ and $a_{j}^{-1}$ lie in the same half-plane, hence they can be deformed out of $P$ until they do not intersect $a_{i}$ at all. In cases (2) and (3), these segments lie in different halfplanes, hence the intersection product equals \pm 1 .

In case (2) the intersection is positively oriented, in case (3) it is negatively oriented, as one sees easily (compare the standard presentation where the word $\left.W\left(a_{1}, \ldots, a_{2 g}\right)=a_{1} a_{2} a_{1}^{-1} a_{2}^{-1} \ldots\right)$. 
Example 6.1. Consider the Fermat elliptic curve $E$ of affine equation

$$
y^{3}=x(x-1) .
$$

Here, $n=3$ and $k=1$, and we have generators

$$
\delta_{0,1}=\gamma_{1} \gamma_{0}^{-1}, \delta_{1,1}=\gamma_{0} \gamma_{1} \gamma_{0}^{-2}, \delta_{2,1}=\gamma_{0}^{2} \gamma_{1},
$$

satisfying

$$
\delta_{0,1} \delta_{1,1} \delta_{2,1}=1, \delta_{1,1} \delta_{0,1} \delta_{2,1}=1 .
$$

Eliminating $\delta_{2,1}$, we get generators $\delta_{0,1} \delta_{1,1}$ with relation

$$
\delta_{0,1} \delta_{1,1}=\delta_{1,1} \delta_{0,1},
$$

as fully expected.

Consider now the example of the curve $C$ of affine equation

$$
y^{3}=x\left(x^{4}-1\right) .
$$

Here, $n=3$ and $k=4$, and we have generators

$$
\delta_{0, j}, \delta_{1, j}, \delta_{2, j}, j=1,2,3,4,
$$

satisfying relations

$$
\begin{gathered}
\delta_{0, j} \delta_{1, j} \delta_{2, j}=1 \\
\delta_{1,{ }_{1}} \delta_{1,{ }_{2}} \delta_{1,{ }_{3}} \delta_{1,4} \delta_{2,1} \ldots \delta_{2,4} \delta_{0,1} \ldots \delta_{0,4}=1 .
\end{gathered}
$$

Set

$$
\delta_{1, j}=: a_{j}, \delta_{2, j}=: b_{j} .
$$

Then we have 8 generators satisfying the relation

$$
a_{1} a_{2} a_{3} a_{4} b_{1} b_{2} b_{3} b_{4} b_{1}^{-1} a_{1}^{-1} b_{2}^{-1} a_{2}^{-1} b_{3}^{-1} a_{3}^{-1} b_{4}^{-1} a_{4}^{-1}=1 .
$$

Using the rule we just described, we find (verify once more) :

$$
a_{i} a_{j}=a_{i} b_{j}=b_{i} b_{j}=1,1 \leq i<j \leq 4,
$$

$b_{1} a_{j}=0, j=1,2,3,4, b_{2} a_{j}=0, j=2,3,4, b_{3} a_{j}=0, j=3,4, a_{4} b_{4}=0$.

Remark 6.2. Together with Jong Hae Keum, Matthew Stover and Domingo Toledo, we proved that the Jacobian $J(C)$ of the above curve $C$ is isomorphic to $E^{4}$, the product of four copies of the Fermat elliptic curve; and we also disovered other curves whose Jacobian is a product (or is isogenous to a product) of elliptic curves.

For the above curve $C$, however, we found later that the same example had been described by Ryo Nakajima in [Nak07.

Acknowledgements: I would like to thank Andreas Demleitner, Jong Hae Keum, Matthew Stover and Domingo Toledo for useful conversations. 


\section{REFERENCES}

[Apo70] Tom M. Apostol: Resultants of cyclotomic polynomials. Proc. Amer. Math. Soc. 24 (1970), 457-462.

[BdF08] Giuseppe Bagnera, Michele de Franchis: Le superficie algebriche le quali ammettono una rappresentazione parametrica mediante funzioni iperellittiche di due argomenti, Mem. di Mat. e di Fis. Soc. It. Sc. (3) 15, 253-343 (1908).

[BCF15] Ingrid Bauer, Fabrizio Catanese, Davide Frapporti: Generalized Burniat type surfaces and Bagnera-de Franchis varieties. J. Math. Sci. Univ. Tokyo 22 (2015), no. 1, 55-111

[Bieb11] Ludwig BieBERBACH: Über die Bewegungsgruppen der euklidischen Räume. (Erste Abhandlung.) Math. Ann. 70, 297-336 (1911).

[Bieb12] Ludwig BieBerBACH: Über die Bewegungsgruppen der euklidischen Räume. (Zweite Abhandlung.) Die Gruppen mit einem endlichen Fundamentalbereich. Math. Ann. 72, 400-412 (1912).

[CamZha05] Frederic CAmpana, QI Zhang: Compact Kähler threefolds of $\pi_{1}$ general type. Recent progress in arithmetic and algebraic geometry, 1-12, Contemp. Math., 386, Amer. Math. Soc., Providence, RI, (2005).

[CatCil93] Fabrizio Catanese, Ciro Ciliberto: On the irregularity of cyclic coverings of algebraic surfaces. Geometry of complex projective varieties (Cetraro, 1990), 89-115, Sem. Conf., 9, Mediterranean, Rende, (1993).

[Cat08] Fabrizio Catanese: Differentiable and deformation type of algebraic surfaces, real and symplectic structures. CIME Course: Symplectic 4manifolds and algebraic surfaces, Springer Lecture Notes in Math. 1938 (2008) 55-167.

[CC17] Fabrizio Catanese, Pietro Corvaja: Teichmüller spaces of generalized hyperelliptic manifolds. Complex and symplectic geometry, 39-49, Springer INdAM Ser., 21, Springer, Cham (2017).

[Cat15] Fabrizio Catanese: Topological methods in moduli theory, Bull. Math. Sci. 5 (2015), no. 3, 287-449.

[CD17] Fabrizio Catanese, Andreas Demleitner: Rigid Group Actions on Complex Tori are Projective (after Ekedahl), arXiv:1711.05545, with an appendix by Benôit Claudon, Fabrizio Catanese, Andreas Demleitner.

[CD18] Fabrizio Catanese, Andreas Demleitner: Hyperelliptic Threefolds with group $D_{4}$, the Dihedral group of order 8, arXiv:1805.01835.

[CD18-2] Fabrizio Catanese, Andreas Demleitner: The classification of Hyperelliptic threefolds, arXiv:1812.09754.

[CHK13] Benoît Claudon, Andreas Höring, János Kollár: Algebraic varieties with quasi-projective universal cover. J. Reine Angew. Math. 679, 207-221 (2013).

[DHS08] Karel Dekimpe, Marek Hąenda, Andrzej Szczepański: Kähler flat manifolds. J. Math. Soc. Japan 61 (2009), no. 2, 363-377.

[Dem16] Andreas Demleitner: Classification of Bagnera-de Franchis Varieties in Small Dimensions, arXiv:1604.07678, to appear in the Annales de la Fac. Sci. de Toulouse.

[Died40] Fritz-Erdmann Diederichsen: über die Ausreduktion ganzzahliger Gruppendarstellungen bei arithmetischer Äquivalenz. Abh. Math. Sem. Hansischen Univ. 13 (1940), 357-412.

[Dres12] Gregory Dresden: Resultants of cyclotomic polynomials. Rocky Mountain Journal of Math. Vol. 42, Number 5, (2012), 1461-1469. 
[EnrSev09] Federigo Enriques, Francesco Severi Mémoire sur les surfaces hyperelliptiques. Acta Math. 32 (1909), no. 1, 283-392, and Acta Math. 33 (1910), no. 1, 321-403.

[Fu88] AkIRA FujIKI: Finite automorphism groups of complex tori of dimension two. Publ. Res. Inst. Math. Sci., 24 (1988), 1-97.

[Kod66] Kuniniko KodaiRA: On the structure of compact complex analytic surfaces. II. Amer. J. Math. 88 (1966) 682-721.

[Lang] Serge Lang: Algebra, Revised third Edition, Springer G.T.M. (2005).

[La01] Herbert Lange: Hyperelliptic varieties. Tohoku Math. J. (2) 53 (2001), no. $4,491-510$.

[Lehm30] Emma Lehmer: A numerical function applied to cyclotomy. Bull. Amer. Math. Soc. 36 (1930), 291-298.

[MKS] Wilhelm Magnus, Abraham Karrass, Donald Solitar: Combinatorial group Theory. Second revised Edition, Dover (1976).

[Miln71] John Willard Milnor: Introduction to algebraic K-theory. Annals of Mathematics Studies 72, Princeton University Press, Princeton (1972).

[Nak07] Ryo Nakajima: On splitting of certain Jacobian varieties. J. Math. Kyoto Univ. 47-2 (2007), 391-415.

[UY76] KoJi Uchida, Hisao Yoshinara: Discontinuous groups of affine transformations of $\mathbb{C}^{3}$. Tohoku Math. J. (2) 28 (1976), no. 1, 89-94.

Lehrstuhl Mathematik ViII, Mathematisches Institut der Universität BAyreuth, NW II, Universitätsstr. 30, 95447 BAyreuth

E-mail address: Fabrizio.Catanese@uni-bayreuth.de 